\title{
Joint Channel and Time Delay Estimation for LTE Positioning Reference Signals
}

\author{
José A. del Peral-Rosado, José A. López-Salcedo \\ and Gonzalo Seco-Granados \\ Universitat Autònoma de Barcelona (UAB) \\ Bellaterra, Spain \\ Email: $\{$ JoseAntonio.DelPeral, Jose.Salcedo, \\ Gonzalo.Seco\}@uab.cat
}

\author{
Francesca Zanier \\ and Massimo Crisci \\ European Space Agency (ESA) \\ Noordwijk, The Netherlands \\ Email: \{Francesca.Zanier, Massimo.Crisci\}@esa.int
}

\begin{abstract}
The ranging performance of the Long Term Evolution (LTE) positioning reference signal (PRS) is enhanced with respect to traditional correlation-based approaches in multipath channels. For that purpose, a joint maximum likelihood (ML) channel and time delay estimation is introduced for the PRS signal. The estimation can be implemented by using the leastsquares (LS) criterion that benefits from the multicarrier flexible waveform. Preliminary results are shown with a comparison of the root-mean-square error (RMSE) of this ML estimator and the corresponding Cramér-Rao Bound (CRB) expression for a specific urban pedestrian channel model.
\end{abstract}

\section{INTRODUCTION}

Localization and positioning systems are every day more important in mobile devices. Their introduction in smart phones has paved the way to deploy location-based services (LBS), such as navigation, advertising or social media, and allowed the location identification of emergency calls driven by legal mandates, such as the E911 in US or the E112 in Europe. Most positioning applications are based on the Global Navigation Satellite Systems (GNSS). However, the robustness of mass-market GNSS receivers is compromised in challenging environments, such as indoor or urban scenarios. Thus, complementary systems are usually proposed to assist the operation of GNSS systems.

The Long Term Evolution (LTE) technology is a promising candidate to complement GNSS positioning services. The LTE standard, specified by the $3^{\text {rd }}$ Generation Partnership Project (3GPP) consortium [1], has defined a dedicated downlink signal for Observed Time Difference of Arrival (OTDoA) positioning, i.e. the positioning reference signal (PRS). The LTE PRS ranging performance has been studied, for instance, by Medbo et al. in [2] with measurements of a channel campaign, by Gentner et al. in [3] for a specific indoor scenario, and from the theoretical and statistical point of view in [4]-[6]. The main advantage of this downlink signal is its waveform, defined by a multicarrier Orthogonal Frequency Division Multiplexing (OFDM) modulation. The multicarrier signals are widely adopted in wireless communications systems because of its flexibility, spectral efficiency and robustness against frequency-selective fading introduced by multipath, among other advantages with respect to traditional singlecarrier signals. These advantages have led to the proposal of multicarrier signals for next-generation GNSS, such as in [7][11]. Nevertheless, the flexibility of multicarrier signals has not been exploited by navigation systems to compensate the effect of multipath in harsh environments.

The aim of this paper is to take advantage of the multicarrier flexibility to jointly estimate the channel and the time delay of the LTE PRS signal, by means of the maximum likelihood (ML) estimator. The multicarrier signal appears to be ideal for this purpose, because the least-squares (LS) criterion can be easily applied to estimate both parameters in the frequency domain. The joint ML estimator is well-known in the literature, and has been applied for array processing in [12], multiuser applications with CDMA signals in [13], and OFDMA uplink synchronisation in [14]. More recently, a joint channel estimation and data detection approach has been implemented in [15] to reduce the multipath interference in cellular networks, but only using the preamble of the multicarrier signal. Thus, further research is necessary to explore the capabilities of the multicarrier signal in order to enhance the time-of-arrival estimation in harsh environments with dense multipath.

Our contribution proposes the further study of this multicarrier capability by showing the ranging performance improvement in multipath channels using the joint ML estimator for the LTE PRS signal. In Sec. II, a description of the LTE standard is presented. Then, the typical channel models are introduced in Sec. III, and the signal model is defined in Sec. IV. An ML correlation-based estimator and the joint ML estimator proposed for time delay estimation are introduced in V. Preliminary results are shown for a static scenario in Sec. VI. Finally, we draw the conclusions in Sec. VII.

\section{LONG TERM EvOlution (LTE)}

Long Term Evolution (LTE) moves towards the fourth generation (4G) of mobile communications. Most of its standard, which is driven by $3 \mathrm{GPP}$, has been inherited from the Universal Mobile Telecommunication System (UMTS) in order to maintain backward compatibility. The main new features introduced are the downlink Orthogonal Frequency-Division Multiple Access (OFDMA) and the Multiple Input Multiple Output (MIMO) data transmission. The signal bandwidth is 
scalable from $1.4 \mathrm{MHz}$ to $20 \mathrm{MHz}$ with a symbol period $T_{s}$ of $66.67 \mu \mathrm{s}$, which corresponds to a subcarrier spacing $F_{s c}$ of $15 \mathrm{kHz}$.

According to the LTE specification [16], the downlink positioning procedure, defined by the OTDoA method, uses the difference in the arrival times of downlink radio signals from multiple base stations (i.e. eNodeBs) to compute the user position. The method relies on a network-based strategy because the eNodeB locations are not provided to the user. First, the user equipment (UE) request assistance information to proceed with the timing measurements. Then, the LTE Positioning Protocol (LLP) transfers the UE measurements to the location server, E-SMLC (Enhanced Serving Mobile Location Center). Based on the UE measurements, the E-SMLC estimates the UE position using a trilateration technique, and this position information is finally sent back to the user.

Regarding to the physical layer, the LTE standard [17] specifies a set of downlink signals based on an OFDM modulation with different time-frequency distributions, whose basic structure is shown in Fig. 1 and 2. Downlink synchronization and reference signals are completely known (like the pilot signals in GNSS), and thus they are suitable for ranging purposes. Especially, the primary and secondary synchronization signal (i.e. PSS and SSS), as well as the cell-specific reference signal (CRS), can be used for signals of opportunity (SoO) applications because they do not require any assistance data. However, LTE follows the typical frequency reuse factor of a cellular network, which is equal to one. Thus, the received serving cell signal interferes with the received neighbour cell signals producing inter-cell interference, and resulting in the near-far effect. In order to obtain proper ranging measurements of the neighbour cells, the LTE standard in Release 9 specifies a positioning reference signal (PRS) that is especially dedicated for positioning purposes and mitigates the near-far effect, due to a higher frequency reuse factor (i.e. of six), by shifting one subcarrier position the frequency pilot allocation transmitted by each base station. The main parameters for PRS configuration are shown in Table I. The PRS signal is scattered in time and frequency in the so-called positioning occasion, which allocates consecutive positioning subframes with a certain periodicity. The sophistication of this signal is even higher when the network mutes the PRS transmissions of certain base stations (i.e. PRS muting), in order to further reduce the inter-cell interference.

\section{Typical Channel Models}

Propagation channel models are essential tools for simulation and testing of wireless transmission systems. The literature is extensive on this topic, and many standards have recommended channel models for specific propagation environments. These models may characterize path-loss attenuation, shadowing and multipath effects. Our interest is focused on the multipath propagation conditions present in typical LTE channels, and their impact on the time delay estimation of the PRS signal.

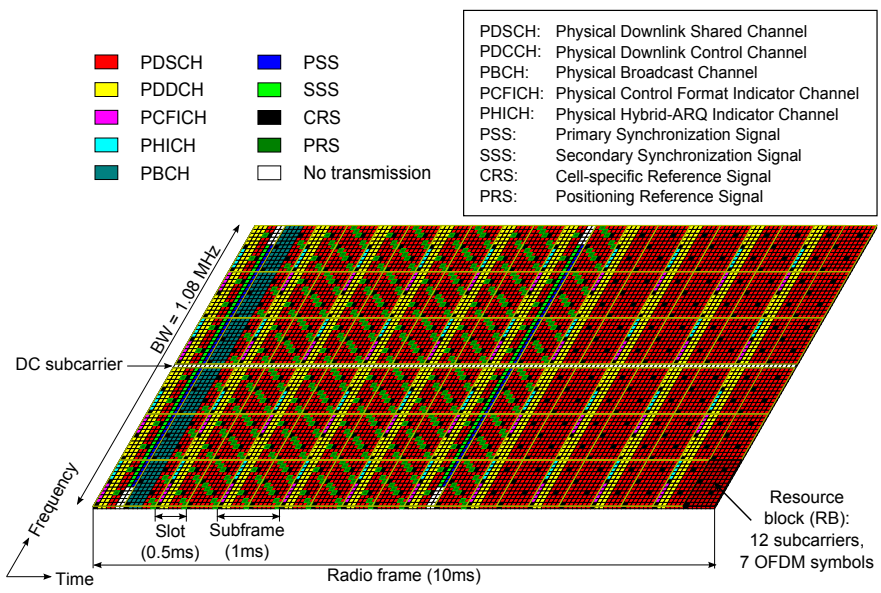

Fig. 1. Time-frequency grid of the LTE signals for $1.4 \mathrm{MHz}$ bandwidth, FDD structure and normal cyclic prefix (CP).

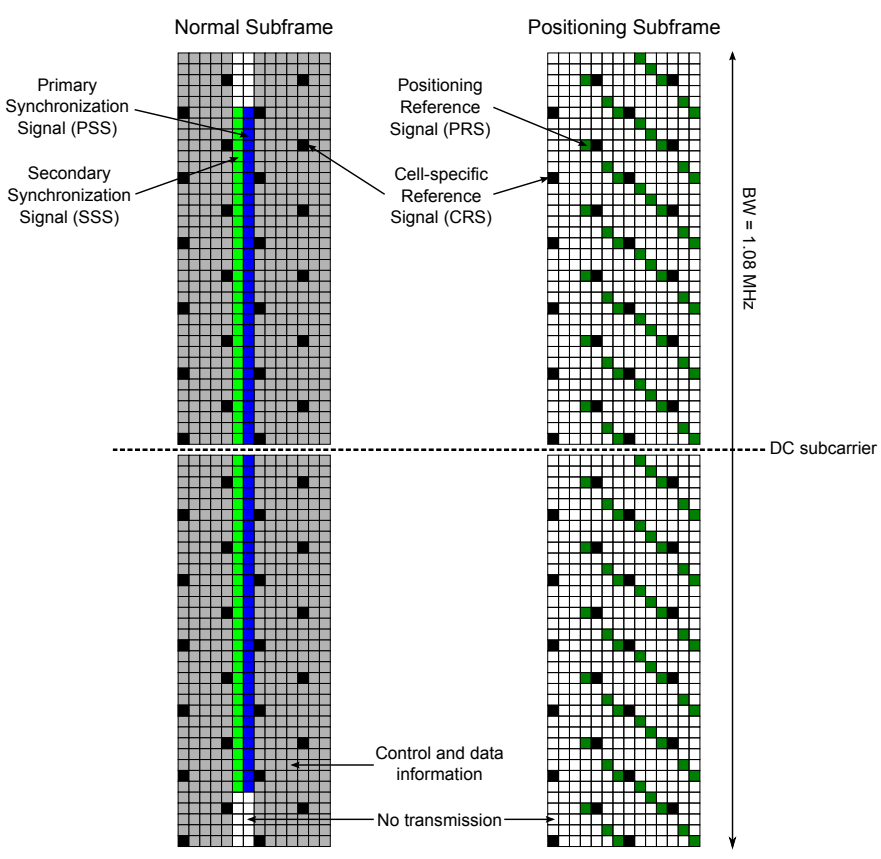

Fig. 2. Example of normal and positioning subframes with 6 resource blocks (RB) for one base station, FDD structure and normal cyclic prefix (CP).

TABLE I

MAIN PARAMETERS OF THE PRS SIGNAL.

\begin{tabular}{ll}
\hline PRS bandwidth & $1.4,3,5,10,15$ and $20 \mathrm{MHz}$ \\
PRS periodicity & $160,320,640$ or $1280 \mathrm{~ms}$ \\
Consecutive subframes $^{1}$ & $1,2,4$, or 6 \\
PRS muting information & 2, 4, 8, 16 bits \\
PRS pattern & 6-reuse in frequency \\
PRS sequence & Length-31 Gold sequence \\
\hline
\end{tabular}

${ }^{1}$ Number of positioning occasion configured for PRS muting (i.e. bit equal to 0 when PRS is muted). 


\section{A. Tapped-Delay Line (TDL) Channel Models}

The LTE standard adopts models based on the ITU-R M.1225 [18] recommendation and the 3GPP TS 05.05 [19] specification for GSM, widely used in the context of third generation mobile systems. The ITU and 3GPP models are defined by tapped-delay line (TDL) models, where each tap corresponds to a multipath signal characterized by a fixed delay, relative average power and Doppler spectrum. Their channel impulse response (CIR) is defined as

$$
h(\tau ; t)=\sum_{l=1}^{L} h_{l} \delta\left(\tau-\tau_{l}\right),
$$

where $L$ is the number of taps, $\tau_{l}$ is the tap delay relative to the first tap, and $h_{l}$ is the Rayleigh-distributed complex channel coefficient for the $l$-th tap, which follows a classical Jakes Doppler spectrum $S(f)$,

$$
S(f) \propto \sqrt{\frac{1}{1-\left(f / f_{D}\right)^{2}}}, \quad \text { for } f \in\left[-f_{D}, f_{D}\right],
$$

being $f_{D}$ the maximum Doppler shift. Considering the highest speed to be supported in LTE as $v=500 \mathrm{~km} / \mathrm{h}$ [20] and a carrier frequency $f_{c}=2 \mathrm{GHz}$, the maximum Doppler shift expected is $f_{D}=f_{c} \cdot v / c \simeq 927 \mathrm{~Hz}$, being $c$ the speed of light. Thus, the $50 \%$ coherence time is computed using Clarke's model [21], and results in

$$
T_{\text {coh }, 50 \%}=\sqrt{\frac{9}{16 \pi}} \cdot \frac{1}{f_{D}} \simeq 0.46 \mathrm{~ms} .
$$

Particularly, the 3GPP consortium agreed, in [22], on the use of the Pedestrian A and Vehicular A channels from [18], and the Typical Urban (TU) channel from [19], in order to model three reference environments characterized by a low, medium and large delay spread, respectively. Nevertheless, they were designed for a $5 \mathrm{MHz}$ operating bandwidth, and an apparent periodicity appears in their frequency correlation properties for higher bandwidths [23]. Thus, the LTE standard has adopted since 2007 an extension of the ITU and 3GPP models by following the procedure described in [23], resulting in the Extended Pedestrian A (EPA), Extended Vehicular A (EVA) and Extended Typical Urban (ETU) channel models. The main parameters of these models, i.e. tap delay $\tau$ and signal-to-multipath ratio (SMR), are specified in Annex B of TS 36.101 [24] and TS 36.104 [25], and shown in Table II. These specifications also define maximum Doppler shifts for each model to represent low, medium and high mobile conditions. Finally, the TDL models can be applied to multiple antenna schemes by introducing spatial correlation matrices, as it is discussed in [26], resulting on a simple LTE MIMO channel model.

\section{B. Geometric-Based Stochastic Channel Models (GSCM)}

The LTE channel can also be modelled with geometricbased stochastic channel models (GSCM). These are more complex models based on the geometry between base station, mobile station and scatterers, following a stochastic
TABLE II

TAPPED-DELAY LINE CHANNEL MODELS PARAMETERS

\begin{tabular}{ccccccc}
\hline \multirow{2}{*}{$\begin{array}{c}\text { Tap } \\
\text { no. }\end{array}$} & \multicolumn{2}{c}{ EPA channel } & \multicolumn{2}{c}{ EVA channel } & \multicolumn{2}{c}{ ETU channel } \\
\hline 1 & 0 & 0.0 & 0 & 0.0 & 0 & -1.0 \\
2 & 30 & -1.0 & 30 & -1.5 & 50 & -1.0 \\
3 & 70 & -2.0 & 150 & -1.4 & 120 & -1.0 \\
4 & 90 & -3.0 & 310 & -3.6 & 200 & 0.0 \\
5 & 110 & -8.0 & 370 & -0.6 & 230 & 0.0 \\
6 & 190 & -17.2 & 710 & -9.1 & 500 & 0.0 \\
7 & 410 & -20.8 & 1090 & -7.0 & 1600 & -3.0 \\
8 & & & 1730 & -12.0 & 2300 & -5.0 \\
9 & & & 2510 & -16.9 & 5000 & -7.0 \\
\hline
\end{tabular}

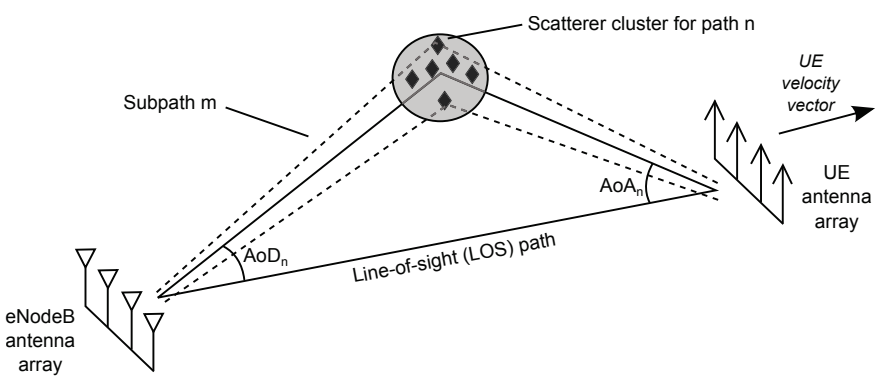

Fig. 3. Scheme of the geometry-based stochastic channel model.

construction. The GSCM models are widely adopted for MIMO channel modelling, e.g. COST 259 channel model [27], COST 273 channel model [28], COST 2100 channel model [29], 3GPP spatial channel model (SCM) [30], or the WINNER channel model [31]. Indeed, the ITU-R M.21351 [32] recommendation for the evaluation of IMT-Advanced systems is based on the WINNER channel model, which is able to operate on bandwidths from $5 \mathrm{MHz}$ to $100 \mathrm{MHz}$. According to this recommendation, the deployment scenarios are classified as indoor hotspot, urban micro-cell, urban macrocell and rural macro-cell. Depending on the scenario selected, large-scale parameters, such as delay spread, angle spread or shadow fading, are randomly generated following the distributions specified in Table A1-7 of [32]. Then, the small-scale parameters, such as delay, power, Angle-of-Arrival (AoA) and Angle-of-Departure (AoD), are randomly distributed for each cluster of propagation rays (i.e. rays with similar delay and directions). Both large- and small-scale parameters are fixed during each channel segment (i.e. the so-called drop). Finally, the time-variant channel realisations of a drop are generated according to the random initial phases of the scatterers. A scheme of the GSCM channel model is shown in Fig. 3.

\section{Limitations of Current Channel Models}

The TDL and GSCM models represent the most typical channel models of current literature. Nevertheless, they are designed for communications purposes, and do not cover important features for positioning applications. First, the time 
delay offset between the base station and the user is not considered in these models, thus the bias produced in non-lineof-sight (NLoS) conditions is not modelled. And second, the channel coefficients are not coherently time-continuous, thus the time evolution of the channel impulse response (CIR) is not completely implemented. Recently, extensions of the WINNER channel model are being studied to jointly model satellite and terrestrial scenarios including these missing features, such as the quasi deterministic radio channel generator (QuaDRiGa) described in [33] and developed under ESA MIMOSA activity (see [34]), or as proposed by Wang et al. in [35].

\section{Signal Model}

Let us define the OFDM baseband signal format for one symbol used in the LTE downlink (without CP) as

$$
x[n]=\sqrt{\frac{2 \cdot C}{N_{c}}} \sum_{k \in \mathcal{N}_{a}} p_{k} \cdot d_{k} \cdot \exp \left(j \frac{2 \pi n k}{N_{c}}\right),
$$

where $C$ is the power of the band-pass signal, $N_{c}$ is the number of subcarriers (excluding unused DC subcarrier), $\mathcal{N}_{a}$ is the subset of active pilot subcarriers $N_{a}$, which must satisfy $N_{a} \leq$ $N_{c}, d_{k}$ are the symbols, and $p_{k}^{2}$ is the relative power weight of subcarrier $k$, which is constrained by $\sum_{k} p_{k}^{2}=N_{c}$ to give the nominal signal power $C$. Considering no interferences and no clock offsets, the received signal is expressed as

$$
r[n]=x[n] * h[n]+w[n],
$$

where $h[n]$ is the channel impulse response (CIR), and $w[n]$ is the additive white Gaussian noise (AWGN). The model of $h[n]$ is defined by the so-called "virtual channel model", according to Bajwa et al. in [36], which is a discrete approximation of the continuous-time channel with a sampling period dictated by the channel bandwidth. As it is described by Hanzo et al. in $[37$, p.25], $h[n]$ can also be called Sampled-Spaced CIR (SS-CIR) and is based on the following channel model

$$
h(t)=\sum_{l=0}^{L-1} h_{l} \delta\left(t-l T_{c}-\tau_{d}\right),
$$

where $L$ is the number of discrete multipath components, $T_{c}$ is the system sampling period, which is $T_{c}=T_{s} / N_{c}, h_{l}$ is the complex channel coefficient for the $l$-th path, and $\tau_{d}$ is the timing offset. The received signal may also be written in the frequency domain, as it is shown by Larsen et al. in [38], with the following matrix notation using an $N$-point discrete Fourier transform (DFT)

$$
\mathbf{r}=\mathbf{B} \boldsymbol{\Gamma}_{\tau_{d}} \mathbf{F}_{L} \mathbf{h}+\mathbf{w}
$$

where

$$
\begin{aligned}
\mathbf{r} & =[r[-N / 2+1], \ldots, r[N / 2]]^{T}, \\
\mathbf{\Gamma} & =\operatorname{diag}\left(e^{j \frac{2 \pi}{T_{s}}(-N / 2+1) \tau_{d}}, \ldots, e^{j \frac{2 \pi}{T_{s}}(N / 2) \tau_{d}}\right), \\
\mathbf{B} & =\operatorname{diag}\left(b_{-N / 2+1}, \ldots, b_{N / 2}\right), \\
\mathbf{h} & =\left[h_{0}, \ldots, h_{L-1}\right]^{T}, \\
\mathbf{w} & =[w[-N / 2+1], \ldots, w[N / 2]]^{T},
\end{aligned}
$$

being $b_{k}=p_{k} \cdot d_{k}$, and $\mathbf{F}_{L}$ is composed of the first $L$ columns of the zero-frequency-centered $N \times N$ DFT matrix,

$$
\mathbf{F}_{L}=\frac{1}{\sqrt{N}}\left[\begin{array}{cccc}
1 & \omega^{-\frac{N-1}{2}} & \cdots & \omega^{-\frac{N-1}{2}(L-1)} \\
\vdots & \vdots & \ddots & \vdots \\
1 & 1 & \cdots & 1 \\
1 & \omega & \cdots & \omega^{L-1} \\
\vdots & \vdots & \ddots & \vdots \\
1 & \omega^{\frac{N-1}{2}} & \cdots & \omega^{\frac{N-1}{2}(L-1)}
\end{array}\right]
$$

where $\omega=e^{j \frac{2 \pi}{N}}$ is a primitive $N$-th root of unity in $j=\sqrt{-1}$.

\section{Time Delay Estimation}

\section{A. Correlation-Based Estimator for AWGN Channel}

As it is well-known, the maximum likelihood estimation (MLE) for the AWGN channel is based on the correlation of the received signal $r[n]$ with a shifted and conjugated version of the reference signal $x[n]$, which is assumed periodical (i.e. circular correlation), in order to find the correlation peak. The resulting correlation between the received and the transmitted signal is defined by

$$
R_{r x}\left(\tau_{d}\right) \doteq \sum_{n=0}^{N_{c}-1} r[n] \cdot x_{c}^{*}\left[n+n_{\tau_{d}}\right]
$$

where $x_{c}[n]$ is a circular shifted version of the original $x[n]$, resulting in the matched filter of the OFDM signal, whose estimated delay is expressed as

$$
\hat{\tau}_{d}=\frac{T_{s}}{N_{c}} \arg \max _{\tau_{d}}\left\{\left|R_{r x}\left(\tau_{d}\right)\right|^{2}\right\},
$$

where $\tau_{d}$ is the time delay in seconds. Thus, the maximum likelihood estimate for the AWGN channel is obtained by measuring the time delay corresponding to the maximum of the correlation function. In Fig. 4, the autocorrelation function of $x_{c}[n]$ is shown for different bandwidth configurations of the LTE positioning reference signal (PRS) using only one OFDM symbol. As it can be noticed, the bandwidth is denoted according to the number of resource blocks (RB) occupied by the PRS signal in the frequency domain (i.e. $180 \mathrm{kHz}$ per RB).

Since the OFDM signal described in (4) is completely known, the Cramér-Rao Bound (CRB) expression for time delay estimation, $\hat{\tau}_{d}$, applied to the LTE signal formats, and disregarding the presence of $\mathrm{CP}$, can be derived from the general definition given by Kay [39] considering a flat fading channel,

$$
\mathrm{CRB}_{\tau_{d}, \text { flat }}=\frac{T_{s}^{2}}{8 \pi^{2} \cdot \mathrm{SNR} \cdot \sum_{k \in \mathcal{N}_{a}} p_{k}^{2} \cdot k^{2}}
$$

where the signal-to-noise ratio is $\mathrm{SNR}=\left(C / N_{0}\right) / B$, being $C / N_{0}$ the carrier-to-noise-density ratio and $B$ the bandwidth of the signal. 


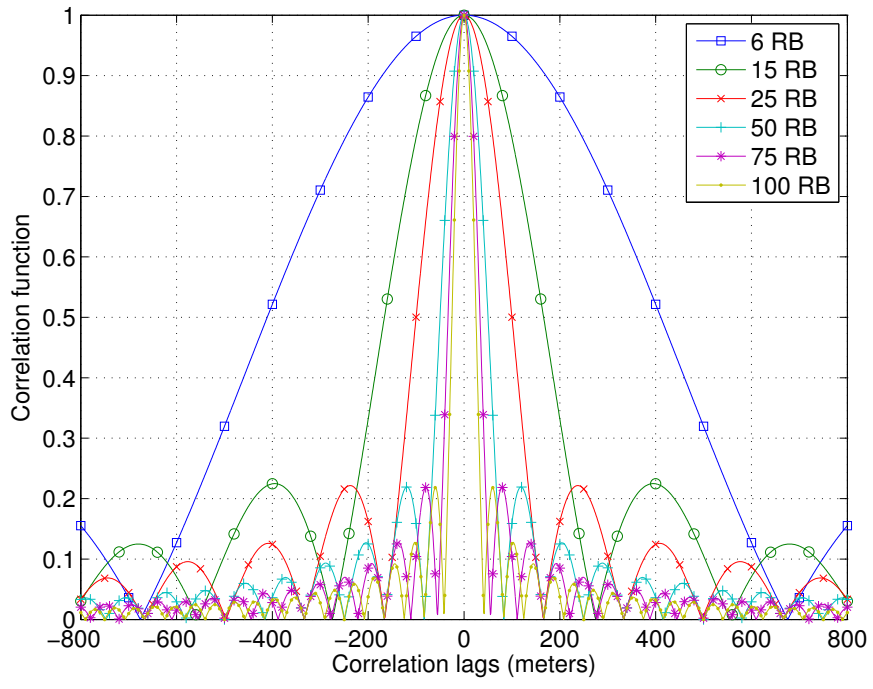

Fig. 4. Autocorrelation function of the LTE PRS signal for the different standard bandwidth and one OFDM symbol.

\section{B. Time Delay Estimator for Multipath Channels Using Joint Maximum Likelihood Channel and Time Delay Estimation}

The presence of multipath channels must be taken into account in the time delay estimation (TDE) process. Thus, our proposal is to aid the TDE estimation by means of the estimation of the channel. For that purpose, the maximum likelihood (ML) estimator of both channel and time delay is used in this paper, as it is derived by Ziskind and Wax in [12]. Following Lilleberg et al. [13], the ML estimator for channel and delay is given by

$$
\left[\begin{array}{c}
\hat{\tau}_{d} \\
\hat{\mathbf{h}}
\end{array}\right]=\arg \min _{\tau_{d}, \mathbf{h}}\left\{\left\|\mathbf{x}-\mathbf{B} \boldsymbol{\Gamma}_{\tau_{d}} \mathbf{F}_{L_{c}} \mathbf{h}\right\|^{2}\right\},
$$

where $L_{c}$ is the true dimension of the channel model. Since we assume that channel and time delay are unknown, the design parameter $L$ is defined to bound the number of taps of the channel. Let us define, the matrix $\mathbf{A}$ function of $\tau_{d}$ and dimensions $N \times L$ as,

$$
\mathbf{A}_{\tau_{d}}=\mathbf{B} \boldsymbol{\Gamma}_{\tau_{d}} \mathbf{F}_{L}
$$

the estimation of the channel response using the least squares (LS) criterion for the pilot subcarriers is found as

$$
\hat{\mathbf{h}}=\left(\mathbf{A}_{\tau_{d}}^{H} \mathbf{A}_{\tau_{d}}\right)^{\dagger} \mathbf{A}_{\tau_{d}}^{H} \mathbf{x},
$$

where $\mathbf{A}^{\dagger}$ denotes the Moore-Penrose generalized pseudo inverse of $\mathbf{A}$, being $H$ the Hermitian conjugate. As it can be noticed, the estimation of $\hat{\tau}_{d}$ and $\hat{\mathbf{h}}$ is decoupled, and the ML delay estimator results in

$$
\begin{aligned}
\hat{\tau}_{d} & =\arg \min _{\tau_{d}}\left\{\min _{\mathbf{h}}\left\|\mathbf{x}-\mathbf{A}_{\tau_{d}} \mathbf{h}\right\|^{2}\right\}= \\
& =\arg \min _{\tau_{d}}\left\{\left\|\mathbf{x}-\mathbf{A}_{\tau_{d}}\left(\mathbf{A}_{\tau_{d}}^{H} \mathbf{A}_{\tau_{d}}\right)^{\dagger} \mathbf{A}_{\tau_{d}}^{H} \mathbf{x}\right\|^{2}\right\}= \\
& =\arg \min _{\tau_{d}}\left\{\left\|\mathbf{P}_{\mathbf{A}, \tau_{\mathbf{d}}}^{\perp} \mathbf{x}\right\|^{2}\right\} \\
& =\arg \max _{\tau_{d}}\left\{\left\|\mathbf{P}_{\mathbf{A}, \tau_{\mathbf{d}}} \mathbf{x}\right\|^{2}\right\},
\end{aligned}
$$

where $\mathbf{P}_{\mathbf{A}, \tau_{d}}=\mathbf{A}_{\tau_{d}}\left(\mathbf{A}_{\tau_{d}}^{H} \mathbf{A}_{\tau_{d}}\right)^{\dagger} \mathbf{A}_{\tau_{d}}^{H}$ is the projection matrix and $\mathbf{P}_{\mathbf{A}, \tau_{d}}^{\perp}=\mathbf{I}-\mathbf{P}_{\mathbf{A}, \tau_{d}}$ is the complement projection matrix. The ML estimator of (23) is computed numerically by maximizing the cost function of $\left\|\mathbf{P}_{\mathbf{A}, \tau_{d}} \mathbf{x}\right\|^{2}$ as a function of $\tau_{d}$. For the case of $L$ equal to one, this joint ML estimator reduces to the traditional correlation-based estimator, as it is derived in [12]. The computational burden of the ML estimator can be alleviated with iterative methods, such as the Alternating Projection (AP) algorithm proposed in [12].

In order to assess the results of the estimator, the CRB for joint estimation can be used as a reference, as in [38], which is defined as

$$
\mathrm{CRB}_{\tau_{d}, \text { oint }}=\frac{\sigma_{n}^{2}}{2} \gamma_{\tau_{d}}^{-1}
$$

where

$$
\begin{aligned}
\gamma_{\tau_{d}} & =\mathbf{h}^{H} \mathbf{F}_{L}^{H} \mathbf{B}^{H} \mathbf{D} \Pi_{\mathbf{B}_{L}}^{\perp} \mathbf{D B} \mathbf{F}_{L} \mathbf{h}, \\
\mathbf{D} & =\frac{2 \pi}{T_{c}} \operatorname{diag}(-N / 2+1, \ldots, N / 2), \\
\mathbf{\Pi}_{\mathbf{B}_{L}}^{\perp} & =\mathbf{I}-\mathbf{B F}_{L}\left(\mathbf{F}_{L}^{H} \mathbf{B}^{H} \mathbf{B} \mathbf{F}_{L}\right)^{-1} \mathbf{F}_{L}^{H} \mathbf{B}^{H} .
\end{aligned}
$$

\section{Vi. Preliminary Results}

Time-of-arrival (ToA) positioning systems are usually implemented with classical correlation-based techniques, such as the delay-locked loop (DLL). Indeed, DLL architectures have low complexity and are optimal for the AWGN channel. However, the presence of multipath rays disturb their ranging performance, producing bias on their time delay estimation. In this section, the maximum likelihood estimator for both channel and time delay is compared with the traditional correlationbased estimator, considering the Cramér-Rao Bound (CRB) as a reference.

\section{A. Use of Typical Channel Models}

In order to ease the assessment of the channel estimation, a typical channel model is sampled to obtain the channel coefficients $h_{l}$ of (6). For that purpose, the multipath rays are defined by sinc functions of a specific bandwidth using the complex channel coefficients $h_{l}$ and time delays $\tau_{l}$ of the channel model. Then, the overall contribution of the rays is obtained by adding the resulting sinc functions. Finally, the translated complex channel coefficients $h_{l}$ are obtained by sampling the overall channel response at $T_{c}$ periods. As it is shown in Fig. 5, an example realisation of the 9-taps ETU channel model is sampled to obtain the complex channel coefficients at $T_{c}$ periods.

\section{B. RMSE of the Joint ML Estimator}

The estimators described in Sec. V are assessed for a static scenario and different signal-to-noise ratio (SNR) levels. Following the procedure of the previous section, the channel impulse response used for this static scenario results in

$$
\begin{aligned}
\mathbf{h}_{0}= & {[1.10-j 0.37,0.47-j 0.26,0.12-j 0.15,} \\
& 0.15-j 0.06,-0.07+j 0.02,0.01+j 0.03],
\end{aligned}
$$




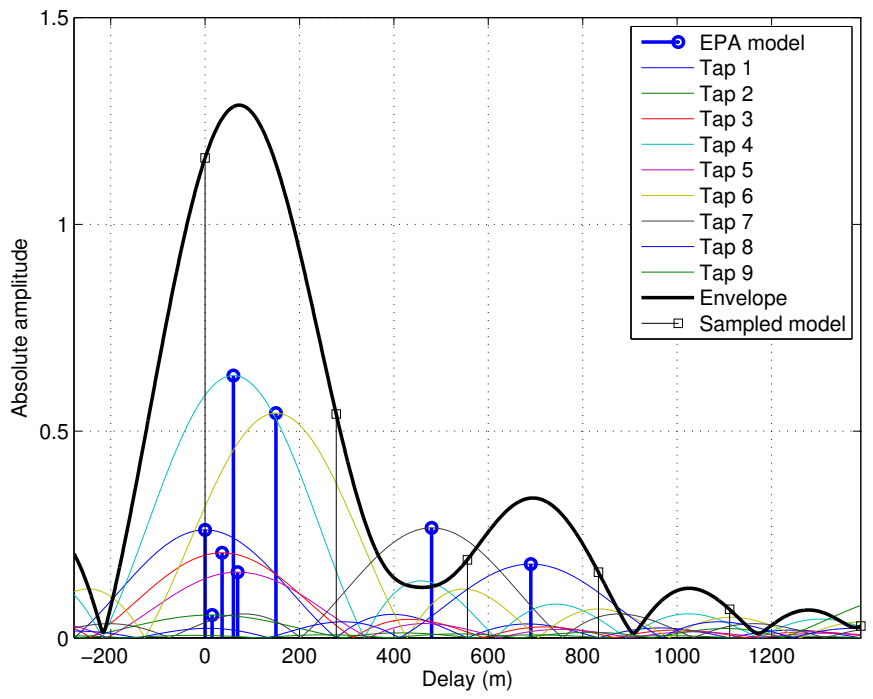

Fig. 5. Sampled version of the ETU channel model using $6 \mathrm{RB}(1.08 \mathrm{MHz})$.

which has been obtained using the ETU channel model for a bandwidth of $1.08 \mathrm{MHz}$ or six resource blocks. The first case of interest is based on considering only the presence of the line-of-sight (LoS) ray. Thus, only the first tap of $\mathbf{h}_{0}$ is convolved with the LTE transmitted signal. Then, the received signal is obtained by adding AWGN noise with SNR values between -25 and $25 \mathrm{~dB}$. Using 1000 Monte-carlo simulations, the root-mean-square error (RMSE) of the joint ML estimator and the matched filter are compared with the CRB considering h, i.e. $\mathrm{CRB}_{\tau_{d} \text {, joint }}$. As it is shown in Fig. 6(a), setting the design parameter $L$ to one, the joint ML estimator attains the CRB bound as well as the matched filter. However, if we consider multipath rays, i.e. the first three taps of $\mathbf{h}_{0}$ are used, $\mathrm{CRB}_{\tau_{d}, \text { joint }}$ is only attained by the RMSE of the joint ML estimator for high SNR, while the matched filter has a bias of 46 meters, as it can be seen in Fig. 6(b). The bias of the matched filter can be computed in absence of noise with the cost function of the joint ML estimator for $L$ equal to one, as it is shown in Fig. 7. Analysing the cost function when setting $L$ to three, one can notice that the optimization problem provides two similar solutions shown with two peaks. This is because for those two estimated delays the absolute value of the estimated channel is equal. In order to avoid this ambiguity, a discrimination criterion is defined based on the assumption that the first channel coefficient $h_{0}$ has the maximum absolute value of $\mathbf{h}$. Thus, the maximum of the cost function is limited to those regions that fulfil the criterion, as it is shown in the example of Fig. 7. Using this criterion, the joint ML estimator attains the CRB for joint estimation, $\mathrm{CRB}_{\tau_{d}, \text { joint }}$, even for low SNR, as it can be seen in Fig. 6(c). The robustness of the estimator could be enhanced by modifying the assumption and introducing a threshold for the minimum magnitude level of the first tap $h_{0}$. Further research is required to investigate the performance of the joint ML estimation in non-line-of-sight (NLoS) conditions.

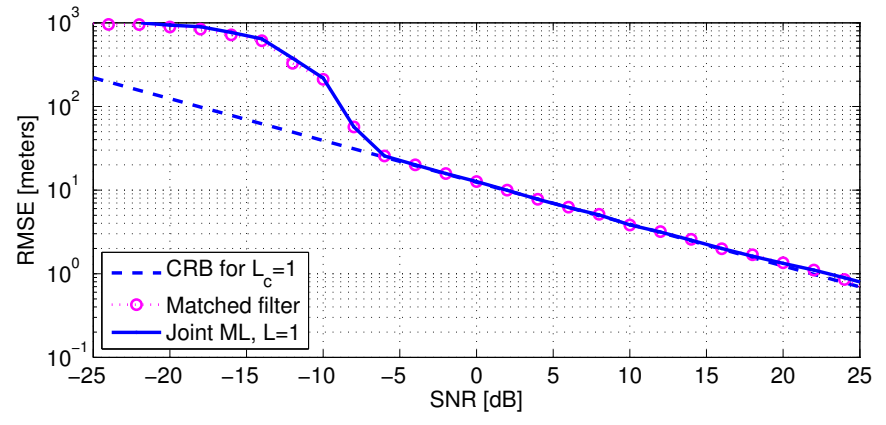

(a) $L_{c}=1$

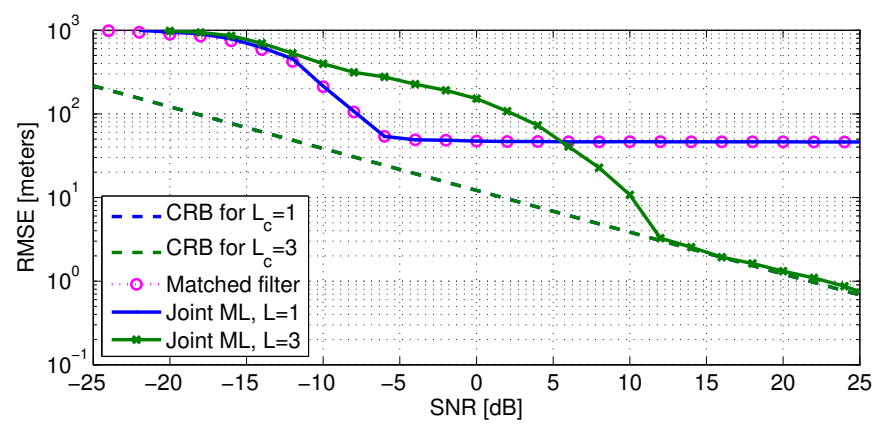

(b) $L_{c}=3$

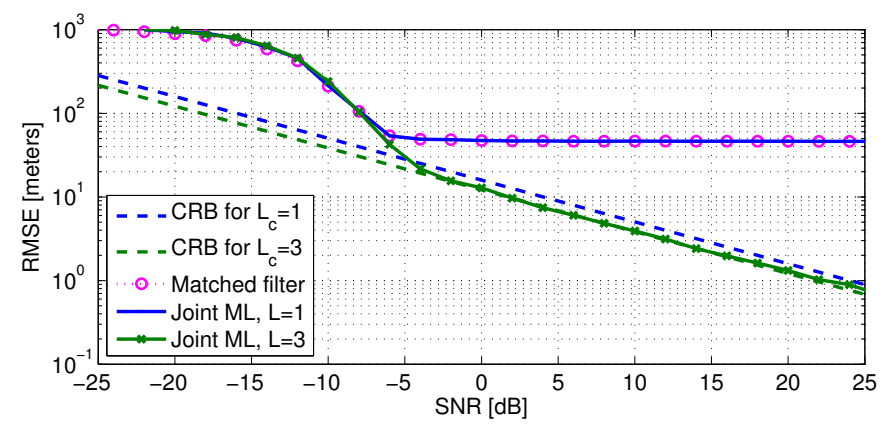

(c) $L_{c}=3$, with discriminator criterion

Fig. 6. Comparison between the joint channel and delay ML estimator and the matched filter using an LTE PRS signal of 6 RB.

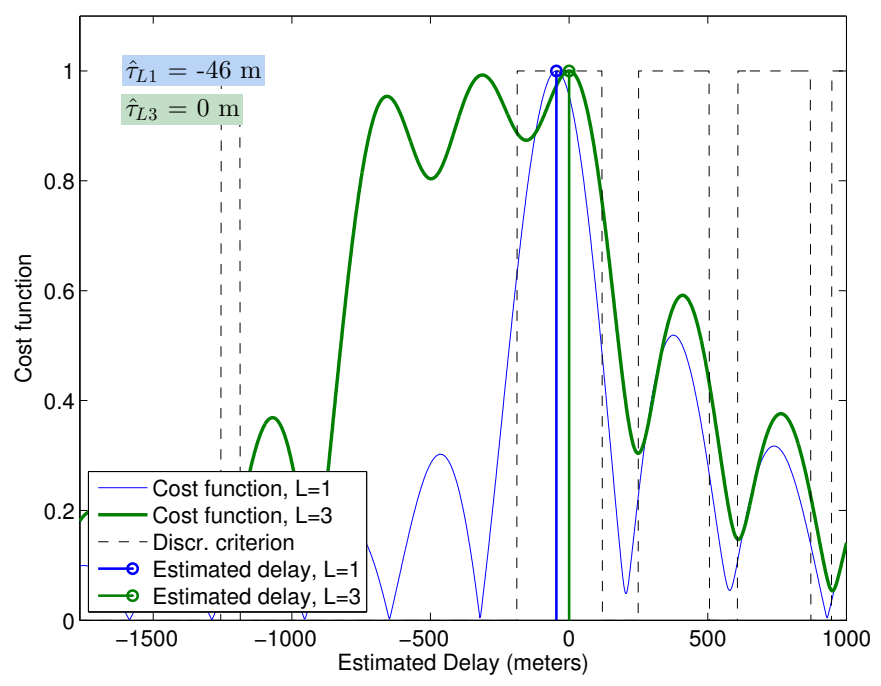

Fig. 7. Cost function using an LTE PRS signal of 6 RB. 


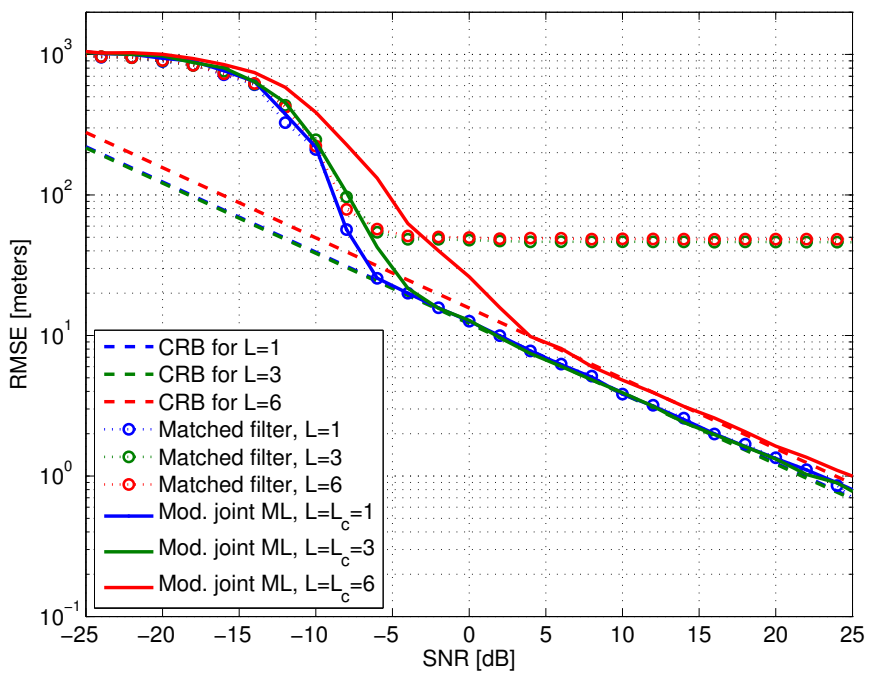

Fig. 8. Comparison of the classical and new approaches when $L=L_{c}$, using an LTE PRS signal of 6 RB.

Finally, the joint ML estimator is implemented with the discriminator criterion to estimate the time delay using the same number of channel coefficients as the true channel, i.e. $L=L_{c}$. As it is shown in Fig. 8, the resulting RMSE attains the $\mathrm{CRB}_{\tau_{d} \text {, joint }}$ for the cases of using $L$ equal to one, three and six. In contrast to the matched filter, the joint ML estimator is unbiased when the channel is perfectly estimated. Further evaluation of the proposed estimator is required to identify those conditions that may introduce a bias on the estimation.

\section{CONCLUSION}

A joint channel and time delay estimator is proposed to exploit the flexibility advantages provided by the multicarrier waveform adopted in the Long Term Evolution (LTE) standard, i.e. the Orthogonal Frequency Division Multiplexing (OFDM) modulation. This estimator is based on the maximum likelihood (ML) estimation, which can be easily implemented in the frequency domain with the least squares (LS) criterion. Using the LTE positioning reference signal (PRS) signal for the lowest bandwidth (i.e. $1.08 \mathrm{MHz}$ ), preliminary results show that the proposed joint ML estimator is unbiased and attains the Cramér-Rao Bound (CRB) for joint estimation in a static scenario, in contrast to the biased-behaviour of traditional correlation-based techniques (e.g. matched filter). Future work is required to further investigate the potential of the proposed estimator, particularly in harsh conditions that may induce a biased estimation.

\section{ACKNOWLEDGMENT}

The content of the present article reflects solely the authors view and by no means represents the official European Space Agency (ESA) view. This work was supported by the ESA under the PRESTIGE programme ESA-P-2010-TEC-ETN-01 and by the Spanish Ministry of Science and Innovation project TEC 2011-28219.

\section{REFERENCES}

[1] 3GPP consortium, home page. [Online]. Available: http://www.3GPP.org

[2] J. Medbo, I. Siomina, A. Kangas, and J. Furuskog, "Propagation channel impact on LTE positioning accuracy: A study based on real measurements of observed time difference of arrival," in Proc. IEEE PIMRC'09, Sep. 2009, pp. 2213 -2217.

[3] C. Gentner, E. Muñoz, M. Khider, E. Staudinger, S. Sand, and A. Dammann, "Particle filter based positioning with 3GPP-LTE in indoor environments," in Proc. IEEE/ION PLANS'12 , Apr. 2012, pp. 301-308.

[4] J. A. Del Peral-Rosado, J. A. López-Salcedo, G. Seco-Granados, F. Zanier, and M. Crisci, "Achievable localization performance accuracy of the positioning reference signal of 3GPP LTE," in Proc. ICL-GNSS, Jun. 2012.

[5] — "Evaluation of the LTE positioning capabilities under typical multipath channels," in Proc. ASMS/SPSC'12, Sep. 2012.

[6] _ "Analysis of positioning capabilities of 3GPP LTE," in Proc. ION GNSS, Sep. 2012.

[7] F. Zanier and M. Luise, "Fundamental issues in time-delay estimation of multicarrier signals with applications to next-generation GNSS," in Proc. 10th Int. Workshop on Signal Processing for Space Communications (SPSC'08), 2008, pp. 1-8.

[8] L. Dai, Z. Wang, J. Wang, and Z. Yang, "Positioning with OFDM signals for the next-generation GNSS," IEEE Trans. on Consumer Electronics, vol. 56, no. 2, pp. 374-379, 2010.

[9] M. Vergara, F. Antreich, M. Meurer, and G. Seco-Granados, "Spreading code design for a MC-CDMA based GNSS pilot signal," in Proc. 5th ESA Workshop on Satellite Navigation Technologies and European Workshop on GNSS Signals and Signal Processing (NAVITEC'10), 2010.

[10] A. Emmanuele, M. Luise, J. Won, D. Fontanella, M. Paonni, B. Eissfeller, F. Zanier, and G. Lopez-Risueno, "Evaluation of filtered multitone (FMT) technology for future satellite navigation use," in Proc. ION GNSS, 2011, pp. 3743-3755.

[11] D. Wang, M. Fattouche, and F. Ghannouchi, "Multicarrier code for the next-generation GPS," EURASIP Journal on Wireless Communications and Networking, no. 1, p. 185, 2012.

[12] I. Ziskind and M. Wax, "Maximum likelihood localization of multiple sources by alternating projection," IEEE Trans. on Acoustics, Speech and Signal Processing, vol. 36, no. 10, pp. 1553-1560, 1988.

[13] J. Lilleberg, E. Nieminen, and M. Latva-Aho, "Blind iterative multiuser delay estimator for CDMA," in Proc. IEEE PIMRC'96, vol. 2, Oct. 1996, pp. 565-568.

[14] M. Pun, M. Morelli, and C. Kuo, "Maximum-likelihood synchronization and channel estimation for OFDMA uplink transmissions," IEEE Trans. on Communications, vol. 54, no. 4, pp. 726-736, 2006.

[15] J. Yang, X. Wang, S. Park, and H. Kim, "Direct path detection using multipath interference cancellation for communication-based positioning system," EURASIP Journal on Advances in Signal Processing, vol. 2012, no. 1 , p. $188,2012$.

[16] 3GPP TS 36.305, Stage 2 functional specification of User Equipment (UE) positioning in E-UTRAN, Std.

[17] 3GPP TS 36.211, Physical Channels and Modulation, Std.

[18] ITU-R M.1225, "Guidelines for evaluation of radio transmission technologies for IMT-2000," 1997.

[19] 3GPP TS 05.05, Radio transmission and reception, Std.

[20] 3GPP TR 25.913, Requirements for Evolved UTRA (E-UTRA) and Evolved UTRAN (E-UTRAN), Std.

[21] T. S. Rappaport, Wireless communications: principles and practice. Upper Saddle River, NJ: Prentice Hall, 2002.

[22] R4-070572, "Proposal for LTE channel models," 3GPP, Ericsson, Nokia, Motorola, Rohde \& Schwarz, RAN4-43, May 2007.

[23] T. Sorensen, P. Mogensen, and F. Frederiksen, "Extension of the ITU channel models for wideband (OFDM) systems," in Proc. IEEE VTC '05, vol. 1, Sep. 2005, pp. 392-396.

[24] 3GPP TS 36.101, UE radio transmission and reception, Std.

[25] 3GPP TS 36.104, BS radio transmission and reception, Std.

[26] R4-060334, "LTE channel models and simulations," 3GPP, Ericsson, Elektrobit, Nokia, Motorola, Siemens, RAN4-38, Feb. 2006.

[27] A. Molisch, H. Asplund, R. Heddergott, M. Steinbauer, and T. Zwick, "The COST259 directional channel model-part i: Overview and methodology," IEEE Trans. on Wireless Communications, vol. 5, no. 12, pp. 3421-3433, Dec. 2006.

[28] L. Correia, Ed., Mobile Broadband Multimedia Networks. Academic Press, 2006. 
[29] R. Verdone, Ed., COST 2100 - Pervasive Mobile and Ambient Wireless Communications. Berlin, Germany: Springer, 2011.

[30] 3GPP TS 25.996, Spatial Channel Model for Multiple Input Multiple Output (MIMO) Simulations, Std

[31] Commission of the European Communities, IST-WINNER II project deliverable D1.1.2, WINNER II Channel Models, Std.

[32] ITU-R M.2135-1 International Telecommunication Union, "Guidelines for evaluation of radio interface technologies for IMT-Advanced," 2008

[33] F. Burkhardt, E. Eberlein, S. Jaeckel, G. Sommerkorn, and R. PrietoCerdeira, "MIMOSA - A joint approach to detailed LMS channel modelling," International Journal of Satellite Communications and Networking, submitted for publication.

[34] E. Eberlein, F. Burkhardt, G. Sommerkorn, S. Jaeckel, and R. PrietoCerdeira, "MIMOSA - Analysis of the MIMO channel for LMS systems," in ESA Workshop on Radiowave Propagation, Dec. 2011.
[35] W. Wang, T. Jost, A. Lehner, and U.-C. Fiebig, "Physical-statistical channel model for joint GNSS and mobile radio based positioning," in Proc. ION GNSS, Sep. 2012.

[36] W. Bajwa, J. Haupt, G. Raz, and R. Nowak, "Compressed channel sensing," in 42nd Annual Conference on Information Sciences and Systems (CISS), 2008, pp. 5-10.

[37] L. Hanzo, Y. Akhtman, L. Wang, and M. Jiang, MIMO-OFDM for LTE, WiFi and WiMAX: Coherent versus Non-coherent and Cooperative Turbo Transceivers. Wiley, 2011, vol. 26.

[38] M. Larsen, G. Seco-Granados, and A. Swindlehurst, "Pilot optimization for time-delay and channel estimation in OFDM systems," in IEEE International Conference on Acoustics, Speech and Signal Processing (ICASSP), May 2011, pp. 3564-3567.

[39] S. Kay, Fundamentals of Statistical Signal Processing: Estimation Theory. Prentice-Hall PTR, 1993-1998. 